\title{
Securely deploying distributed computation systems on peer-to-peer networks
}

\author{
Kobe Vrancken \\ imec-DistriNet, KU Leuven \\ Heverlee, Belgium \\ kobe.vrancken@cs.kuleuven.be
}

\author{
Frank Piessens \\ imec-DistriNet, KU Leuven \\ Heverlee, Belgium \\ frank.piessens@cs.kuleuven.be
}

\author{
Raoul Strackx \\ imec-DistriNet, KU Leuven \\ Heverlee, Belgium \\ raoul.strackx@cs.kuleuven.be
}

\begin{abstract}
More and more off-the-shelf processors support the dynamic construction of Trusted Execution Environments. For instance, Intel Software Guard Extensions (Intel SGX) supports the construction of so-called enclaves on modern Intel Core processors. Hence, it is interesting to design and evaluate practical security architectures that leverage this new technology.

One of the possibilities of this new technology is that it enables deployment of traditional distributed applications that require a group of mutually trusting machines, on top of a group of mutually distrusting machines such as a peer-to-peer network. This paper proposes and evaluates an Intel SGX based approach to securely deploy a subset of distributed systems called distributed computation systems in a peer-to-peer fashion, with strong confidentiality and integrity guarantees and without modification of the original system.

The approach is evaluated by applying it to distcc, a distributed compiler. This result of this process is a new program called p2pcc, a distributed peer-to-peer compiler. We created two different versions of $\mathrm{p} 2 \mathrm{pcc}$. In the first version, any process spawned on one of the untrusted peers runs in its own enclave, thus providing a very fine-grained form of isolation. Our evaluation shows that the performance cost on today's Intel SGX implementation is too high. The second version of p2pcc groups all processes running on behalf of the same user within the same enclave, thus providing coarser isolation, but still providing strong isolation on all security boundaries. Our evaluation shows that the second approach has good performance while providing strong security guarantees even on current SGX processors.

Our results provide evidence that deploying existing distributed computation systems in a peer-to-peer fashion is practical.
\end{abstract}

\section{CCS CONCEPTS}

-Security and privacy $\rightarrow$ Distributed systems security; $\bullet$ Software and its engineering;

\section{KEYWORDS}

Security, peer-to-peer, distributed systems, Intel SGX

Permission to make digital or hard copies of all or part of this work for personal or classroom use is granted without fee provided that copies are not made or distributed for profit or commercial advantage and that copies bear this notice and the full citation on the first page. Copyrights for components of this work owned by others than ACM must be honored. Abstracting with credit is permitted. To copy otherwise, or republish, to post on servers or to redistribute to lists, requires prior specific permission and/or a fee. Request permissions from permissions@acm.org.

SAC '19, April 8-12, 2019, Limassol, Cyprus

(C) 2019 Association for Computing Machinery.

ACM ISBN 978-1-4503-5933-7/19/04 ..\$15.00

https://doi.org/10.1145/3297280.3297315

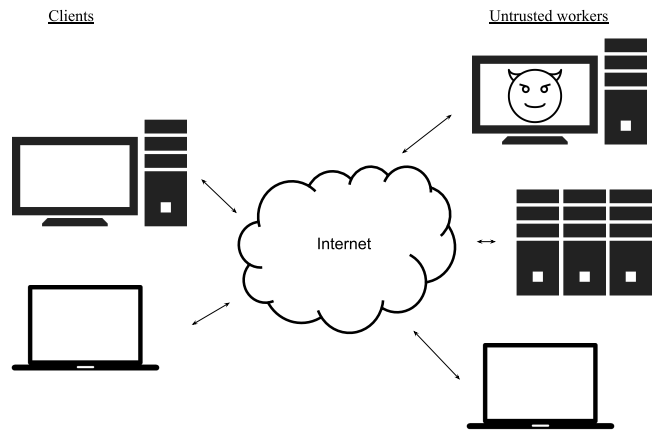

Figure 1: Overview of a peer-to-peer network. Any client can function as a worker and vice-verca.

\section{ACM Reference Format:}

Kobe Vrancken, Frank Piessens, and Raoul Strackx. 2019. Securely deploying distributed computation systems on peer-to-peer networks. In The 34th ACM/SIGAPP Symposium on Applied Computing (SAC '19), April 8-12, 2019, Limassol, Cyprus. ACM, New York, NY, USA, Article 4, 10 pages. https: //doi.org/10.1145/3297280.3297315

\section{INTRODUCTION}

Many desktop-PC's and workstations use only a small fraction of their CPU cycles and memory on average. Only when the workstation user starts a resource intensive task, the full power of the machine [18] is useful to make sure that such tasks complete with acceptable latency. Examples of tasks that exhibit this burst behavior in terms of resource requirements are video editing or image processing (where for instance filtering or compression are resource intensive), and software development (where for instance a full software build is resource intensive).

A useful approach to mitigate these burst resource usages is to distribute the resource intensive task across several machines on the local network. A prototypical example is distcc [32], a distributed $\mathrm{C} / \mathrm{C}++$ compilation system, that distributes the various compilation tasks needed for a full software build across several neighboring machines. ${ }^{1}$

Local distributed computation systems like distcc are very useful to reduce the latency of resource intensive tasks, but they also come with security risks. All of the machines participating in the execution of a task need to be trusted: they can in principle violate the confidentiality and/or integrity of data that is processed (e.g.

\footnotetext{
${ }^{1}$ Another alternative approach (but one that is not discussed in this paper) is to execute such resource intensive tasks in a trusted cloud.[21,35]
} 
source code for compilation). It is possible to mitigate these security risks using Intel SGX, allowing us to use the excess resources of a peer-to-peer network more efficiently[39].

In this paper, we propose an approach to deploy these distributed computation systems without modification on peer-to-peer networks. A peer-to-peer network consists of a collection of untrusted workers, each of which could potentially be malicious (fig 1 ). The network itself could also be malicious. Furthermore, any client in the network can also function as a worker and vice-versa.

We enable the distribution of tasks to other hosts, without the need to trust the users, operating system or software on these hosts. Our design relies on the attestation and isolation properties of Intel SGX $[2,19,30]$ to provide strong confidentiality and integrity guarantees for the distributed computation, even in the presence of malicious peers. Our approach only needs to trust the hardware processors.

We evaluate our approach by applying it to distcc. The resulting compiler, p2pcc, is a distributed compiler that can be deployed in an untrusted peer-to-peer network with strong security guarantees.

In summary, this paper makes the following contributions:

- We propose a generic approach to securely deploy unmodified distributed computation systems on top of untrusted peer-to-peer networks.

- We apply this approach to a case study, distcc, a distributed compiler. This results in p2pcc, which is, to our knowledge, the first distributed compiler that can be securely deployed in a peer-to-peer fashion with both integrity and confidentiality guarantees.

- We implement two different versions of p2pcc that differ in the way they map the different distcc processes onto enclaves. Both implemented versions will be open-sourced with the release of this paper ${ }^{2}$.

- A small but interesting conclusion can also be drawn from the evaluation and comparison of the performance and security of both p2pcc versions, namely that there is a need for an efficient implementation of the fork and exec system calls in Library OS enclaves.

The remainder of this paper is structured as follows: First we clarify the assumed attacker model and the security objectives for peer-to-peer computation systems. Next, Section 3 provides background on both Intel SGX and library operating systems. Section 4 presents our approach. We discuss how it can be applied in practice with the distcc case study in Section 5 . Security and performance evaluation of this case study follows in Section 7. Finally we present related work and conclude.

\section{PROBLEM STATEMENT}

A distributed computation system consists of two parts: (1) the frontend, running on the client machine, and (2) a computation service or daemon, running on all workers that want to make available part of their resources to assist in the computation.

The front-end is responsible for dividing a task in subtasks, distributing these subtasks over available workers, and collecting the results. The computation service accepts tasks over the network, executes them, and sends back the results.

\footnotetext{
${ }^{2}$ https://github.com/KobeVrancken/p2pcc
}

Such a setup works very nicely in an environment where all workers, including their (root-level) users, and system software can be trusted not to maliciously interfere with the execution of subtasks. But an attacker in control of one of the workers can interfere with the distributed computation in unacceptable ways. For instance, such an attacker could inject Trojan code in a software application compiled with distcc.

The problem we set out to address in this paper is to provide a solution for deploying a distributed computation system on an untrusted peer-to-peer network with strong security guarantees and without modification of the original system.

Attacker model. We assume that an attacker is in control of both the network, as well as one or more of the workers running the computation service. The attacker has root-level access to these machines, but we assume that physical attacks against their processors are out of scope.

The client running the front-end is trusted: we assume the attacker has no control over the front-end, or any of the system software running on the host where the front-end is running.

Another class of attacks that we consider out-of-scope for this paper are side-channel attacks. While attackers might learn something about the computations performed by computation services by using a variety of side-channels such as for the sizes of network messages [35], the execution time of the computation [6,14,36], or other observable side-effects of the computations happening [8, 9, 26, 45], we consider protection against such side-channel a separate and orthogonal topic, and we do not counter such attacks in our design. Furthermore we assume that any attack breaking the SGX security objectives are mitigated by future Intel microcode updates. For instance, the powerful Foreshadow attack [44] has been mitigated in this manner.

Security objective. Under such an attacker model, we want to maintain the confidentiality and integrity properties that local execution provides (i.e. execution where all computation happens on the trusted workstation running the front-end). In other words, distributed execution can only have an availability impact on computations, but no integrity or confidentiality impact.

More specifically, with integrity of the computation, we mean that any modification by the attacker to the code or data sent to a computation service or to the result retrieved from this service will be detected and rejected by the client.

With confidentiality of the computation we mean that the attacker cannot read the data being sent to or received from the computation service, neither while in transit nor while it is being computed on.

Lastly, availability is not guaranteed. An attacker might be able to block progress of the computation, for instance by interfering with network communication, by shutting down or rebooting some of the workers under the attacker's control, or by tampering with the code to be executed resulting in rejection of the results by the client.

If the client receives responses from all computation services that tasks were sent to, and if these responses pass all security checks, then the result of the computation is the same as if it would have been executed locally, without attacker intervention. 
Of course, besides the security objective, the proposed system must satisfy other objectives: the secure implementation of a distributed computation system must satisfy the same functional requirements as existing implementations. In addition, we want to quantify the performance cost associated with the stronger security guarantees and it is an objective of our design to keep these costs low.

\section{BACKGROUND}

\subsection{Intel SGX}

Intel Software Guard eXtensions (SGX) $[2,19,30]$ are extensions to the Intel x86 CPU architecture family which allow for the creation of Trusted Execution Environments (TEE) on supported machines. The Trusted Execution Environments provided by Intel SGX are called enclaves. An enclave is an isolated region of memory which can only be accessed by code running inside this memory. When an enclave is running on a system, it provides predefined entry points which are accessible to code running outside of the enclave.

SGX guarantees that a program running inside the enclave will execute exactly as specified even in the presence of a system-level attacker. Furthermore, this isolated piece of software cannot be inspected or altered by any other program running on the same system, no matter what the privilege level of that software is. The integrity and confidentiality of code and data inside of an enclave is guaranteed even in the presence of a hardware attacker.

Intel SGX even protects against some hardware attacks. Enclaved code and data resides in plaintext only within the CPU package. Whenever enclaves are stored in main memory, it is confidentiality, integrity and freshness protected. This makes Intel SGX resistent against cold boot attacks [15] and related, low-cost hardware attacks.

3.1.1 Attestation. Once software is deployed inside of an enclave the integrity and confidentiality of this software is guaranteed. It remains important to verify that the deployed software was not replaced or modified by an attacker while creating the enclave (which is possible). In order to actually communicate with an enclave there also needs to be a mechanism to establish a shared secret with it.

The combination of verifying that an enclave was initialized exactly as specified by the designer and establishing this shared secret is called attestation. When it is done locally between two enclaves the process is called local attestation. When a remote party attests an enclave on a different machine the process is called remote attestation.

During the creation of an enclave, the SGX processor generates a measurement of every added page (MRENCLAVE). This enclave measurement together with a hash of the public key of the enclave creator (MRSIGNER) are used in both local and remote attestation. Local attestation between two enclaves uses a specific processor instruction to deliver these measurements to one another without the possibility of forging them.

Remote attestation is more complicated. The remote processor has no way of verifying the correctness of a received measurement. To implement remote attestation Intel provides each system with a special enclave called the quoting enclave. This enclave has access to a unique signing key inside the processor which is used to sign the generated measurement. Only the quoting enclave has access to this signing key. The signature can eventually be verified by the attestor through the use of the Intel Attestation Service.

The enclave measurements can be used to verify that an enclave was initialized correctly. The attestor is authenticating the enclave using these measurements. Since this provides one-way authentication from the attestor to the attestee it allows them to perform a Diffie-Hellman [10] based protocol and establish a shared secret. If two-way authentication (whereby the enclave authenticates its attestor) is required, it is also possible to embed a public key of the attestor in the memory of the enclave. This public key cannot be altered since this would change the enclave measurement. It can be used to encrypt the Diffie-Hellman messages from the enclave to the attestor.

\subsection{Library OS}

The threat model of SGX considers the operating system untrusted. An enclave program cannot blindly make use of operating system functionality since that could compromise the integrity and confidentiality of the program. System calls are thus disabled inside an enclave. This is an issue when one wants to run unmodified applications inside enclaves.

Haven [4] was the first to come with a solution to this problem by bundling a Library OS together with the unmodified application inside of the enclave. This approach has since been adopted in other work such as SCONE [3], Graphene-SGX [43] and SGX-LKL [33]. Of these systems, SCONE is a closed source commercial product. SGX-LKL at the time of writing only has a public github repository available and have yet to publish their work. Graphene-SGX however is a working, open-source and peer-reviewed system with different research projects already using their system. On top of that, Graphene-SGX is, to the best of our knowledge, the only system supporting exec and fork functionality. For these reasons we will be using Graphene-SGX in our implementation.

\section{APPROACH}

In this section we describe our approach to deploying unmodified distributed computation systems in a practical, transparent way on top of peer-to-peer networks.

\subsection{Problems}

To deploy a legacy distributed system securely on top of a peerto-peer network we need to decide what part of the distributed system will run on untrusted nodes and thus needs to be integrity and confidentiality protected. Secondly, we need a way for all other unprotected code in the distributed system to interact with this protected part in a secure manner.

Current Library OS implementations already allow us to deploy an unmodified application inside an enclave. What is not solved by these systems, however, is how to securely do this for multi-process distributed systems. This paper provides a solution for attestation and secure communication between multiple distributed processes without modification of the original application. Problems such as peer discovery or incentive systems for peers to perform work are left out of scope. 


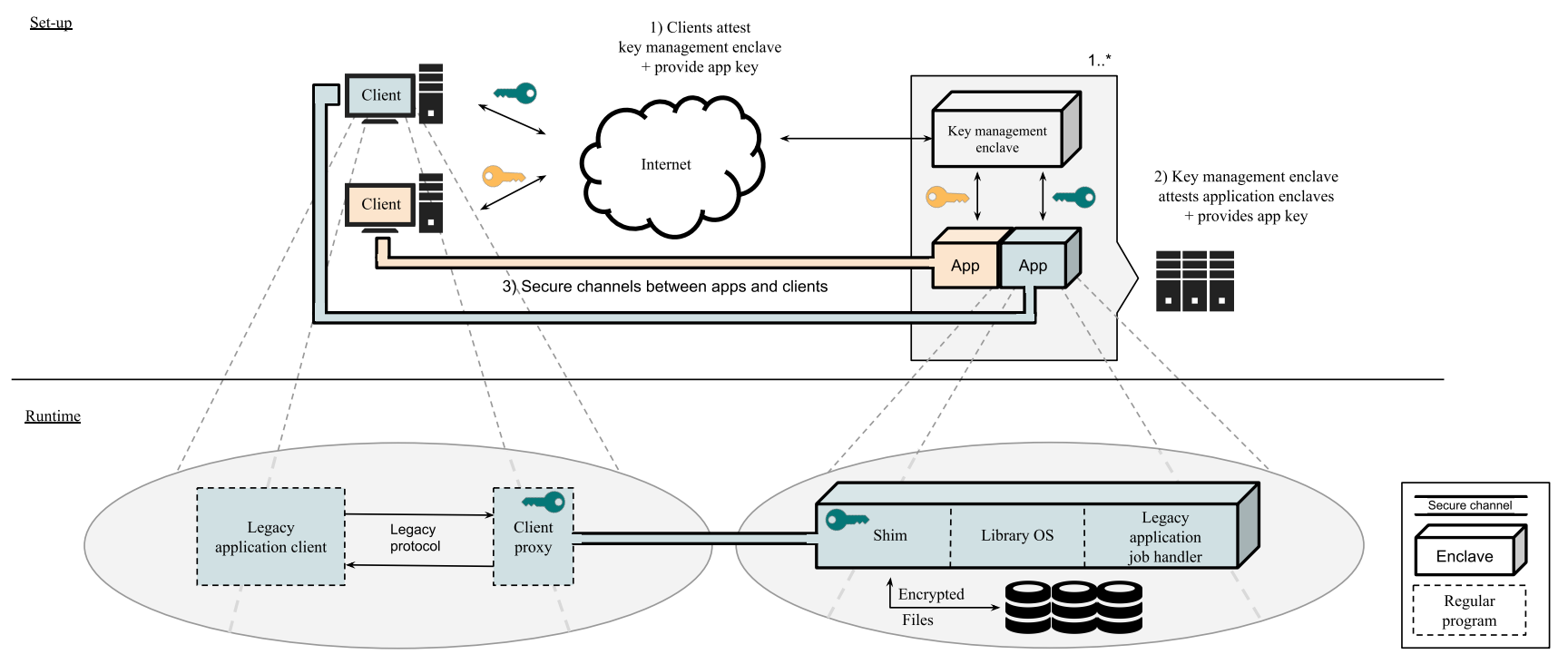

Figure 2: Overview of our solution. The top part shows how a worker in the system is initialized and provisioned with a secret key using the key management enclave. The bottom part zooms in on both the client that uses the computation service and the enclaves on the untrusted workers executing the service.

\subsection{Components}

To deploy the unmodified computational part of the application on top of untrusted nodes we rely on existing Library OSes. This allows us to deploy these parts without needing to modify the original code. However, to allow these protected parts of the system to be deployed and communicate transparently with unmodified unprotected parts in a practical and secure way, we introduce new components.

In order to be practical these components should allow us to first transparently perform attestation of the protected parts of the system and afterwards automatically encrypt the input and output data sent from trusted client machines to these protected parts. Figure 2 shows an overview of the proposed components and how they are used during the set-up and runtime of a distributed computation.

Two of our suggested components are deployed on the untrusted machines:

Key management enclave An enclave that is initially attested by clients and manages their application keys. Multiple stakeholders share this enclave. It can independently attest other enclaves created on the same system and provision them with the correct application key.

I/O shim library A shim library that is dynamically preloaded in Library OS enclaves. It is provisioned with a secret key by the key management enclave after local attestation and uses this to transparently encrypt and decrypt enclave $\mathrm{I} / \mathrm{O}$ operations. Together with the client proxy it transparently establishes a secure communication channel between the worker and the client.

This collection is completed by a single component that runs on the trusted client machine:
Client proxy A program that connects the unmodified legacy client of the distributed computation system to the remotely deployed and protected worker machines. It attests these remote workers by attesting their key management enclave and transparently encrypts and decrypts all communication between them and the unmodified client.

\subsection{Usage}

In this section we will describe in more detail the internals of each of the system components, how they interact with each other and how they can be used to securely deploy a distributed computation system on top of a peer-to-peer network.

To join the workforce of a peer-to-peer distributed computation network, an untrusted node needs to initially load the key management enclave. This is shown in the first step in figure 2. The key management enclave will be used as an entry point of trust for any client that wants to execute a task on this worker machine. Using the remote attestation procedure as described in section 3.1.1, clients attest that the key management enclave was loaded correctly by the untrusted worker. After remote attestation, the key management enclave and the client share a secret in form of a symmetric encryption key. From this point on, the key management enclave can attest new enclaves on the same system using local attestation, and by proxy it can provide trust for the client in these new enclaves. It does this concretely by providing new enclaves with the symmetric encryption key that was also shared with the client (step 2, fig 2). This enables direct secure bidirectional communication between the client to these new enclaves without needing another remote attestation procedure (step 3, fig 2).

In our model of distributed computation systems these new enclaves will contain the parts of the distributed program that are 
needed to execute tasks. They are Library OS enclaves that need to be attested by the key management enclave. However, the code of these programs per the requirements cannot be modified to include attestation code. To achieve this oblivious attestation scheme a shim library is preloaded in the Library OS enclaves, as shown in the runtime section of figure 2 . The shim library contains an entry point to perform local attestation. Furthermore, the shim layer in our implementation transparently intercepts all calls to the GNU Socket API.

Any calls to send and recv are automatically intercepted and encrypted or decrypted. We include a MAC with each message and add a nonce to the plaintext to prevent forgery and replay attacks. File system operations are handled in a similar way. All file system operations are intercepted and mapped directly to the Intel Protected File System API for Linux. This implementation encrypts files using a key derived from a master key (in our case the application key) and the file name. Consequently, all files are integrity-protected using their file name. This means that if the untrusted OS changes the file name it becomes impossible to decrypt the contents of the file. It also means that different versions of a file generated by our application can be interchanged by the operating system if these versions were generated by our application with the same application key and file name. Distcc already chooses random file names for all files used in the compilation process. Thus, one would need to have generated exactly the same random file name with the same application key before a version replacement attack is possible. However, for some other applications that do depend heavily on the file system this can open up powerful attack vectors. The shim library can mitigate these attacks by choosing unique file names for every file it creates with the same key. The file system API can be replaced with a more advanced file system[1,7] which does not suffer from these problems, if necessary.

Thanks to this shim we do not need to modify the original program code nor do we need to modify the Library OS implementation. An alternative to the shim would be to directly modify the Library OS to include local attestation and $\mathrm{I} / \mathrm{O}$ encryption. We chose a less invasive approach, which can be applied to any Library OS that supports dynamic loading ${ }^{3}$.

Workers in the peer-to-peer computation system can thus be initialized by having a key management enclave remotely attested by the client. This enclave by proxy attests the Library OS enclaves which contain part of the unmodified legacy application. The clientside of the application runs in an unprotected domain and should also be oblivious to any attestation or encryption steps. To achieve this, we provide a client proxy, as shown in the runtime section of figure 2. Legacy clients connect and talk to the client proxy instead of connecting directly to the remote workers. The client proxy attests the key management enclaves on the worker nodes and encrypts all communication with these nodes using the established symmetric encryption key. It is used to establish a tunnel between the legacy client and application while remaining oblivious to their internals.

\footnotetext{
${ }^{3}$ Dynamic loading is part of the POSIX standard. It is therefore reasonable for Library OSes to support this feature
}

\section{CASE STUDY: P2PCC}

We illustrate and evaluate our approach by securing distcc [32], a distributed compiler. It is used to speed up compilation time in companies that have spare computing resources. This is done purely for performance reasons without any security measures. All compilation workers therefore have to be trusted. Deploying distcc with our solution results in a new compiler which we named p2pcc. p2pcc is a compiler that can distribute compilation jobs to untrusted SGX-enabled machines while still guaranteeing confidentiality and integrity. This makes it possible to deploy the compiler in a peerto-peer fashion, whereby a group of mutually distrusting people can bundle resources and create a private peer-to-peer compiling cloud.

\subsection{Background: distcc}

distcc consists of two different parts: a front-end and a daemon. The front-end runs at the client side and has the same command line interface as gcc. It can for example be used as the compiler in a Makefile. The daemon is installed on all worker machines that take part in the compilation process. The distcc front-end either forwards compile jobs to systems running the distcc daemon or compiles them locally if local resources are available. The distcc daemon in turn will forward received compilation commands to the compiler installed locally on their machine. In the end each compilation is handled by an actual $\mathrm{C} / \mathrm{C}++$ compiler such as gcc or clang. In our implementations and benchmarks we always use clang as the actual compiler that distcc (or p2pcc) uses.

When a developer compiles a file using the distcc front-end, the front-end checks for available machines in a whitelist of allowed volunteers. A simple load balancing mechanism is used, whereby the first machine in this whitelist that is ready to accept a job will receive it. Members are thus prioritized based on the order in which they appear in this whitelist. Distcc is meant to be used in combination with a parallel build system such as the one provided by GNU Make. The build system analyzes which compile jobs can be executed independently and runs these in parallel. When using distcc as the compiler in a distributed build, these independent compile jobs will be distributed in parallel to the daemons in the whitelist.

Our approach is applied easily to distcc and its components. The distcc front-end runs on the client machine and connects to the network encryption proxy. The distcc daemon is deployed inside a Library OS enclave with an I/O shim, on the worker machine. However, the distcc daemon itself is a multi-process application that calls both the clang front-end and cc1 (which is the compiler process of clang). We are left with two choices on how to map these processes onto enclaves.

A first option is to deploy all these processes in a separate enclave, as shown in figure 3 . The second approach bundles these separate processes in a single enclave, as shown in figure 4 . We implement and evaluate both approaches.

5.1.1 Multi-enclave approach. The first approach maximizes isolation. Figure 3 displays this setup. Distcc spawns a clang process for each compilation unit. This clang process executes the clang front-end, which in turn creates a new process for running the actual cc1 compiler. Thus, for each incoming compilation job the 


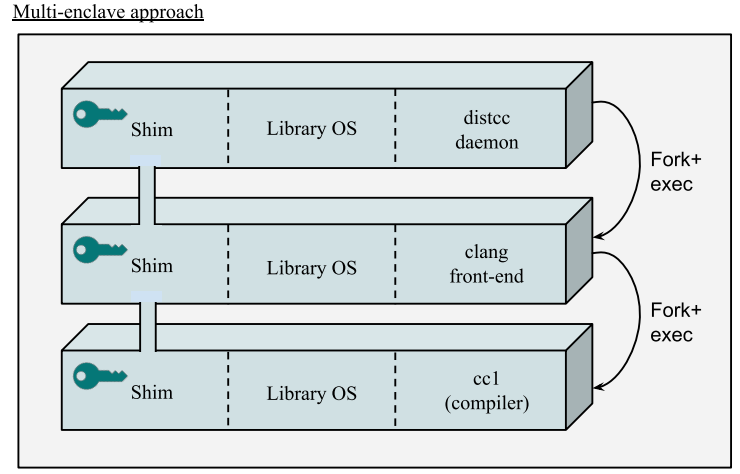

Figure 3: Overview of the multi-enclave approach to deploying a multi-process legacy application.

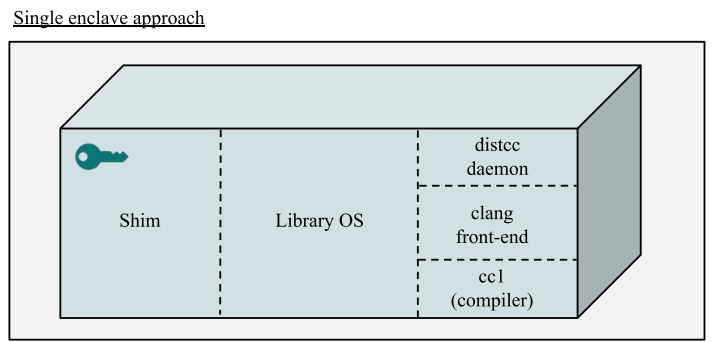

Figure 4: Overview of the single enclave approach to deploying a multi-process legacy application.

distcc daemon spawns two new processes. Each of these processes is placed in its own separate enclave together with a copy of the Library OS and the I/O shim. By relying on the components presented in Section 4 no changes to the legacy processes that are being created, are required.

5.1.2 Single enclave approach. In this second approach, we colocate the multiple processes within the same enclave. As the Library OS now only needs to be instantiated once, pressure on SGX reserved memory is reduced significantly. Since all of the processes belong to the same stakeholder there is no need for them to reside in separate enclaves, and separating them only makes sharing resources more complicated.

Figure 4 displays this setup. The distcc deamon, clang frontend and cc1 compiler are co-located within the same enclave and are running on top of a single Library OS instance. Whenever a compilation unit finishes compilation, its allocated resources are freed. The distcc daemon then prepares to accept a new compilation unit (of the same client).

Unfortunately, at the time of writing there is no library OS that natively supports deploying multiple processes in a single enclave. To evaluate the second approach we implemented it manually by merging the different binaries and replacing fork and exec calls with regular method calls, as we explain in section 6 . One of the
Table 1: Lines of code inside the different enclaves in our system

\begin{tabular}{|c|c|c|c|c|}
\hline & Lang & Vanilla & Added & Total \\
\hline \multicolumn{5}{|c|}{ Normal enclaves } \\
\hline Key management enclave & $\mathrm{C}$ & - & 516 & 516 \\
\hline \multicolumn{5}{|c|}{ Library OS enclaves } \\
\hline Graphene LibOS & $\mathrm{C}$ & 654,486 & - & 654,486 \\
\hline & asm & 138 & - & 138 \\
\hline distcc & $\mathrm{C}$ & 26,015 & - & 26,015 \\
\hline clang/LLVM & $\operatorname{mix}$ & $4 * 10^{\wedge} 6$ & - & $4^{*} 10^{\wedge} 6$ \\
\hline
\end{tabular}

conclusions of this work, is that it is valuable for a Library OS to add support for multiple processes within an enclave. ${ }^{4}$

\section{IMPLEMENTATION}

In this section we will discuss the implementation details of the approaches. Table 1 shows the total lines of code for each of the enclaves. The key management enclave is a very small enclave. This makes it feasible to harden it against attacks, either by writing it in a memory-safe language, formally verifying it or using other attack mitigation mechanisms [37].

The application enclaves in contrast are very large. Both clang and libc (included in the library OS) have large codebases that need to be included inside these enclaves. In the first approach the applications are divided over three enclaves where each enclave at least contains the Graphene LibOS. The second approach includes all of them in a single enclave. The total size of this enclave is close to 5 million lines of code.

The attestation mechanism used is based on the reference implementation provided by the Intel SGX SDK [20]. To establish the shared secret we use an authenticated version of Elliptic Curve Diffie Hellman based on the SIGMA protocol [24]. The EC symmetric key that is established using this protocol has a key size of 128-bit. This key is used to to encrypt messages with AES in GCM mode. The key is also used in the Protected File System as a master key from which filename-based encryption keys are derived. The decision to use these specific cryptographic primitives was made based on the reference implementations in the Intel SGX SDK [20].

In section 5 we claim that the distcc daemon is executed without any modification inside a Graphene-SGX enclave. In practice the distcc daemon executes a system call at start time where it discards the ring-0 privilege level in case it has it. Since this system call has no meaning for enclave programs it was not implemented in Graphene-SGX. We thus had to remove it from the distcc daemon as well ${ }^{5}$. This has no impact on the behaviour or performance of the application.

To run the distcc daemon in an enclave together with clang (for the single enclave approach) we had to merge the clang and distcc daemon binaries into a single one. We did this by compiling distcc

\footnotetext{
${ }^{4}$ Multiple processes within a single enclave need to share a single address space in user mode, making this an impossible feature for mutually distrusting processes. However, mutually trusting processes, such as the different processes spawned by distcc and clang, can share a single address space.

${ }^{5}$ Alternatively, we could have modified Graphene-SGX to intercept and ignore this system call. This would allow us to deploy distccd completely unmodified.
} 
as a library and bundling this library with clang/LLVM. We then made a version of clang which starts LLVM and afterwards calls the old distcc daemon main function. Whenever this main function receives a compilation job it calls the clang compiler main function. Clang itself is a front-end to an assembler, compiler and linker. The front-end has the same command line interface as gcc. However, the internal compiler uses custom flags. A typical clang compilation uses the front-end to convert the command line arguments to internal flags before forking a new process in which the compilation is executed. Instead, we modified the clang front-end to call the main function of the compiler after performing the argument conversion, instead of forking a new process.

\section{EVALUATION}

\subsection{Security Evaluation}

The security (of both versions) relies heavily on the primitives used and the ability to keep the application key secret. We assumed a strong attacker model where the underlying kernel has been compromised. This implies that an attacker may attempt to directly access enclaved code and data. Such attacks are prevented by Intel SGX. In our approach security sensitive data is only passed to enclaves after they attested that they were created correctly. Afterwards each communication channel is protected with a shared cryptographic key.

The key management enclave ensures that enclaves belonging to different stakeholders use unique keys. Multiple stakeholders use this same enclave for key management. It is thus essential that it cannot be exploited. To ensure this the code-base of the key management enclave remains tiny, as shown in table 1 . It is feasible to rewrite it in a memory-safe language preventing lowlevel memory exploits [5, 25, 37] or to formally verify the correctness of the enclave in order to minimize potential attacks.

Applications running within application enclaves, rely on the Graphene library OS. This implies a significant code base that may contain vulnerabilities. However, the interface these enclaves expose is limited. As communication channels to these enclaves are protected, an attacker providing arbitrary messages will not pass the code's integrity check. Hence, while vulnerabilities within this code base are likely, it is challenging for a root-level attacker to exploit them.

An attacker who is able to push her own compilation jobs, on the other hand, is more powerful. By specially crafting source code, vulnerabilities within the compiler may be exploited. This could compromise the enclave and reveal the application key. This in turn would allow her to breach confidentiality and integrity of any messages exchanged. Our solution ensures that different stakeholders will never be co-located within an application enclave which ensures that even that scenario does not breach the security of other stakeholders.

For our p2pcc use case, we implemented two versions. The first places each spawned process in a separate enclave, while the second version bundles them together in a single enclave. The first version exposes a larger interface, since each separate process exposes their own interface to the untrusted part of the system. The second version only exposes the interface of the parent process, in this case the distcc daemon. Exposing a larger interface makes vulnerabilities

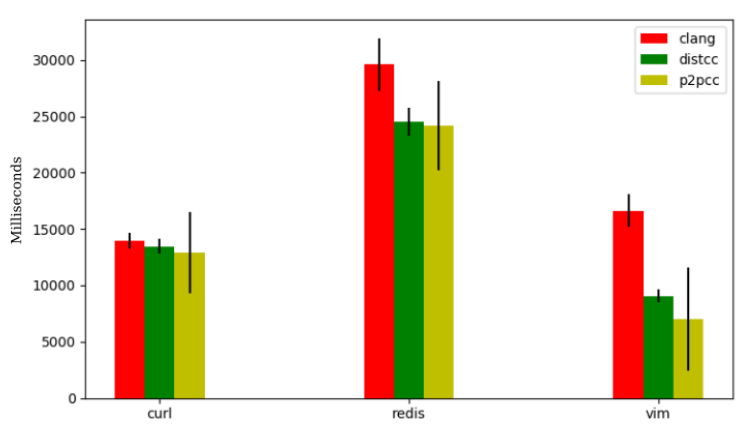

Figure 5: Comparison of mean build times of curl, redis and vim. The projects were built 50 times with each of the compilers with GNU Make. The single enclave version of p2pcc was used for this benchmark. The multi-enclave version is evaluated separately.

Table 2: Average time spent by the multi-enclave version during the different steps needed to compile a single compilation unit.

\begin{tabular}{lr}
\hline Enclave operation & Average time \\
\hline (1) fork distcc daemon & $683 \mathrm{~ms}$ \\
(2) exec clang & $633 \mathrm{~ms}$ \\
(3) fork clang & $810 \mathrm{~ms}$ \\
(4) exec clang compiler & $646 \mathrm{~ms}$ \\
(5) actual compilation & $\Delta$ \\
Total & $\mathbf{2 7 7 2 m s + \Delta}$ \\
\hline
\end{tabular}

more likely to occur since the attacker has a larger variety of ways to interact with the system.

The second version, on the other hand, is more likely to be prone to unintentional application crashes. Indeed, during our implementation we encountered several Graphene bugs and limitations. These are not security sensitive, but a single bug can take down all applications within that enclave, which is undesirable.

\subsection{Performance Evaluation}

To evaluate the performance of our approach we compare the performance of distcc running natively to the performance of both p2pcc implementations.

The performance of each implementation was measured by timing the build process of well-known Linux applications while using each implementation as the dedicated $\mathrm{C} / \mathrm{C}++$ compiler of the build. As a reference we have also included measurements of a fully local build with clang. The projects were chosen from the list of most forked $\mathrm{C} / \mathrm{C}++$ GitHub repositories [13]. From this list we chose projects that can be built using GNU Make. ${ }^{6}$ We excluded projects with external dependencies that are not present on our universitymanaged SGX machines.

\footnotetext{
${ }^{6}$ Due to a resource leak in Graphene-SGX in which file descriptors are not closed correctly we limited ourselves to projects with less than 1024 compilation units.
} 
Our implementations were deployed on a cluster of eleven commodity workstations ${ }^{7}$ supporting Intel SGX. Each build process was invoked from a single build master. From the build master the compilations were distributed over the ten other available workstations.

When using distcc in a practical scenario it is advised to include the local machine in the build process and to only offload work whenever the local machine is at maximum capacity. This ensures that distcc will always offer improved performance over local compilation. However, in order to get an accurate performance comparison between distcc and p2pcc we prevented both of them compiling locally. All work has to be distributed to the SGX-enabled workers. For small projects that can be compiled quickly on a local machine this causes high relative overheads. This limits the advantage of distributing the compilation, as can be seen for curl on figure 5 .

7.2.1 Multi-enclave approach. One of our first observations running the benchmarks was that the first approach, in which each process is separated into their own enclave, suffers from big performance problems. After a single run we measured that a complete build of curl, redis and vim completed in 12, 23 and 15 minutes respectively. From figure 5 we can see that this is around fifty times slower even than local compilation. For this reason we do not include the performance of this approach in figure 5 and instead opt to discuss it separately.

A deeper analysis of the performance problems showed that the majority of the execution time is being spent creating new enclaves. A breakdown of the time spent during the compilation of a single compilation unit is shown in table 2. As explained before, GrapheneSGX creates a new enclave for each call to fork and for each call to exec. To compile a single compilation unit, distcc first (1) forks a new copy of its process and (2) executes clang. In turn (3) clang forks itself and (4) executes the actual compiler cc1. Lastly (5) cc1 compiles the actual compilation unit, which for most compilation units can complete in well under 100 milliseconds.

In total this process creates four different enclaves (step 1 to 4 ). Each of these enclaves are large Graphene-SGX enclaves of at least $256 \mathrm{MiB}$, containing the full glibc library. The entire memory region needs to be cryptographically measured and the different enclaves are contending for only a small region of EPC memory. This causes start-up times in the order of several hundreds of milliseconds.

From the times in table 2 we can see that in order to compile one compilation unit there is a preparation time of almost three seconds, while compiling a compilation unit typically happens in well under 100 milliseconds. This explains the high performance overheads that the first approach exhibits. The authors of the Graphene-SGX acknowledged [43] that fork and exec are slow in the current implementation. In any case, this use case shows that there is a class of applications in which new processes are created in a high frequency which - to the best knowledge of the authors - is not yet supported by any Library OS with acceptable performance. Functionality announced in SGX2, the second version of Intel SGX, might help alleviate these problems[43], as we will explain in section 7.2.3.

\footnotetext{
${ }^{7}$ Dell OptiPlex All in One 7440 with a quad core Intel(R) Core(TM) i5-6500 Skylake CPU @ $3.20 \mathrm{GHz}$
}

7.2.2 Single enclave approach. The results of benchmarking the second approach are shown in figure 5 . In the second approach all processes share a single enclave. There is no need to create a new enclave for each compilation. Figure 5 shows that distcc and this implementation of p2pcc exhibit similar performance. No noticeable differences can be seen from these measurements. The overhead caused by the encryption of messages and the context switches from enclave mode to regular mode are small enough relative to the time it takes to compile a file. From this comparison we can conclude that the second approach allows us to securely deploy distcc on a peer-to-peer network without negatively affecting the performance, even on current hardware.

7.2.3 Discussion on EPC memory size and limitations. SGX reserved memory is limited. In current Intel SGX versions, it only measures $128 \mathrm{MiB}$, of which only $96 \mathrm{MiB}$ can be used to store enclaved code and data. The remainder is used by SGX to store metadata about protected pages. When enclaves grow larger than this limit, or when multiple enclaves are created that together exceed this limit, enclave pages need to be evicted. As this requires pages to be confidentiality, integrity and version protected, this comes at a significant cost in performance.

In our implementation, every single Library OS enclave is larger than this EPC limit. The main reason is that the Library OS itself is large and that it needs enough memory pages to be reserved for its heap. In practice most of these processes use only a small subset of their reserved heap pages, so all others are paged out by the system. In the projects we compiled using our system the compiler did not encounter compilation units that are so large that pages need to be swapped during compilation. If this would occur it would probably trash the performance of the compilation job.

Still we consider this a limitation that is not inherent to this system. Future SGX (or other TEE) systems might allow larger EPCs thereby improving the initialization time of our system and allowing for even these rare extremely heap-intensive compilation jobs. Furthermore SGX2 will allow for dynamic memory management after enclave initialization therefore removing the need to reserve this many heap pages during enclave creation.

\section{RELATED WORK}

Ensuring software executes correctly and in complete isolation has been a challenging problem. Early research endeavors focused on redesigning the operating system itself. This resulted in microkernels that provide strong security guarantees to applications running on top of them. The most notable example is seL4 [27] which was formally verified [22] and MINIX [17, 42] which is currently used in the Intel Management Engine [38].

The biggest downside of microkernels is that they provide a different interface to processes. Legacy applications need to be modified before they can run on different operating systems. To avoid this huge engineering effort, virtual machine monitors have been considered [16]. Instead of porting all applications, a separate virtualized machine can run already ported applications. Legacy applications remain supported by another virtual machine running legacy operating systems.

Memory isolation is only guaranteed while the system is up and running. An attacker able to compromise the system's file system, 
or provide a malicious system update may be able to overwrite the kernel while stored on disk. To defend against such attacks, system software could make use of a Trusted Platform Monitor (TPM). By measuring each binary while the system boots, boot policies can be enforced. For example, only when the system follows the expected load path and no unknown binaries or configuration files are loaded, the system is able to access previously stored data.

The combination of trusted boot and virtual machine monitors raised the question as to what services should be provided to trusted components. Adding additional services increases the likelihood of software vulnerabilities in the "more secure" part. Therefore a tendency developed for ever smaller Trusted Computing Bases [12, 41]. This eventually led to the design of "protected module architectures" (PMAs) where protected modules are only accessible through specific entry points. All other accesses are prevented. The platform provides almost no other services besides memory isolation. Instead protected modules would use platform services such as network connections, but add additional security measures (e.g., TLS). While early designs [29] focused on virtual machine monitors [28, 29], hardware-based PMAs soon followed [23, 31, 34, 40]. With Intel SGX launching with the Skylake microarchitecture in $2015[2,19,30]$, a commercial PMA is now widely available.

Intel SGX sparked wide interest from both academics as well as industry. One of the first prototypes was VC3. By relying on SGX's isolation guarantees, Schuster et al. [35] provide a more secure MapReduce implementation. On one hand code is kept isolated from untrusted servers, while on the other users are guaranteed that the returned result is correct and complete.

While SGX provides strong isolation guarantees to VC3, it does not protect against side-channel attacks. An attacker observing messages exchanged, can infer information about the processed data. To defend against such attacks, Dinh et al. [11] proposed $M^{2} R$. By inserting dummy messages between servers, such side-channels are avoided.

Both VC3 and $\mathrm{M}^{2} \mathrm{R}$ deploy a distributed computation system on a collection of untrusted machines. In contrast with our work, they only allow this specifically for MapReduce-computations and do not support more general unmodified legacy applications.

Another use case of Intel SGX in the space of cloud computing is SecureKeeper [7]. Many distributed applications rely on a coordination services such as ZooKeeper for naming services, configuration management and message exchanges. However, such data is often security sensitive. By combining Intel SGX's security guarantees with ZooKeeper, a service is provided that guarantees the confidentiality and integrity of data handled by ZooKeeper. SecureKeeper is a specific modification of ZooKeeper to support SGX and does not support deploying other unmodified legacy applications.

While SGX originally was intended for small, security critical applications such as one-time password generation or enterprise rights management, software to run legacy applications in SGX enclaves was developed soon after its launch $[3,4,33]$. This enables businesses such as Fortanix to provide security guarantees to cloud users, even in case of a malicious or compromised cloud provider.

While current Library OSes support the deployment of unmodified applications in SGX enclaves they do not attempt to solve this problem for distributed computation systems, which is what we do in this paper.

\section{CONCLUSIONS \& FUTURE WORK}

Distributed computation systems enable large workloads to be easily outsourced. Unfortunately, existing solutions such as distcc require users to place complete trust in all remote platforms. Using Intel SGX such requirements can be significantly reduced.

We proposed a solution and implemented components to allow the deployment of unmodified distributed computation systems on peer-to-peer networks, allowing us to leverage the power of under-utilized systems.

As a use case of our solution, we implemented and evaluated two versions of $\mathrm{p} 2 \mathrm{pcc}$, a distributed compiler based on distcc that can be deployed in a network of untrusted peers. Modern compilers repeatedly spawn new processes, each with a very specific task in the compilation process. For our first version, each of these processes is deployed in their own enclave. While this version of p2pcc achieves to handle such workloads transparently, it does incur significant performance impacts.

In our second version we avoid this overhead by allowing multiple processes of a single stakeholder to share the same enclave. Unfortunately, such support is not yet available in existing Library OSes. After manually implementing the second version of p2pcc by merging the different processes into a single one we managed to create a distributed compiler that can be run on a commodity peer-to-peer network with low performance impact.

From our two implementations of the peer-to-peer compiler we can draw two conclusions for possible future work. Our first implementation shows that there is a need for a more efficient implementation of the fork and exec system calls in existing Library OSes. Since a compiler spawns several processes in a single compilation process, the current cost of several hundreds of milliseconds to create a Library OS enclave is detrimental to the systems performance. Extending the size of the EPC and the adoption of SGX2 could already make this process more efficient.

The high performance of our second implementation shows that it can be useful for multiple processes belonging to the same stakeholder to share a single enclave and Library OS. Sharing an enclave improves performance, manages EPC memory more efficiently and can reduce the size of the interface an attacker might use to exploit the processes that reside inside. In order to support this, we urge the research community to look into new ways of partitioning SGX enclaves into smaller protected regions. Future extensions to SGX (SGX2) allowing for dynamic enclave memory management will make this more feasible.

\section{ACKNOWLEDGEMENTS}

The research presented in this paper was partially supported by the Research Fund KU Leuven, and by a gift from Intel Corporation. Raoul Strackx is supported by a grant of the Research Foundation - Flanders (FWO).

\section{REFERENCES}

[1] Adil Ahmad, Kyungtae Kim, Muhammad Ihsanulhaq Sarfaraz, and Byoungyoung Lee. 2018. OBLIVIATE: A Data Oblivious File System for Intel SGX. (2018).

[2] Ittai Anati, Shay Gueron, S Johnson, and V Scarlata. 2013. Innovative Technology for CPU Based Attestation and Sealing. In Proceedings of the 2nd International Workshop on Hardware and Architectural Support for Security and Privacy (HASP'13), Vol. 13. ACM, New York, NY, USA. 
[3] Sergei Arnautov, Bohdan Trach, Franz Gregor, Thomas Knauth, Andre Martin, Christian Priebe, Joshua Lind, Divya Muthukumaran, Dan O'Keeffe, Mark L. Stillwell, David Goltzsche, David Eyers, Rüdiger Kapitza, Peter Pietzuch, and Christof Fetzer. 2016. SCONE: Secure Linux Containers with Intel SGX. In Proceedings of the 12th USENIX Conference on Operating Systems Design and Implementation (OSDI'16). USENIX Association, Berkeley, CA, USA, 689-703.

[4] Andrew Baumann, Marcus Peinado, and Galen Hunt. 2014. Shielding applications from an untrusted cloud with Haven. In USENIX Symposium on Operating Systems Design and Implementation (OSDI'14).

[5] Andrea Biondo, Mauro Conti, Lucas Davi, Tommaso Frassetto, and Ahmad-Reza Sadeghi. 2018. The Guard's Dilemma: Efficient Code-Reuse Attacks Against Intel \{SGX\}. In Proceedings of the 27th USENIX Conference on Security Symposium. USENIX Association, 1213-1227.

[6] Ferdinand Brasser, Urs Müller, Alexandra Dmitrienko, Kari Kostiainen, Srdjan Capkun, and Ahmad-Reza Sadeghi. 2017. Software Grand Exposure: SGX Cache Attacks Are Practical. CoRR abs/1702.07521 (2017).

[7] Stefan Brenner, Colin Wulf, David Goltzsche, Nico Weichbrodt, Matthias Lorenz, Christof Fetzer, Peter Pietzuch, and Rüdiger Kapitza. 2016. SecureKeeper: Confidential ZooKeeper Using Intel SGX. In Proceedings of the 17th International Middleware Conference (Middleware '16). ACM, New York, NY, USA, Article 14, 13 pages. https://doi.org/10.1145/2988336.2988350

[8] Jo Van Bulck, Nico Weichbrodt, Rüdiger Kapitza, Frank Piessens, and Raoul Strackx. 2017. Telling Your Secrets without Page Faults: Stealthy Page Table-Based Attacks on Enclaved Execution. In 26th USENIX Security Symposium (USENIX Security 17). USENIX Association, Vancouver, BC, 1041-1056.

[9] Guoxing Chen, Sanchuan Chen, Yuan Xiao, Yinqian Zhang, Zhiqiang Lin, and Ten Lai. 2018. SgxPectre Attacks: Leaking Enclave Secrets via Speculative Execution. (Feb. 2018).

[10] Whitfield Diffie and Martin Hellman. 1976. New directions in cryptography. IEEE transactions on Information Theory 22, 6 (1976), 644-654.

[11] Tien Tuan Anh Dinh, Prateek Saxena, Ee-Chien Chang, Beng Chin Ooi, and Chunwang Zhang. 2015. M2R: Enabling Stronger Privacy in MapReduce Computation. In 24th USENIX Security Symposium (USENIX Security 15). USENIX Association, Washington, D.C., 447-462.

[12] T. Garfinkel, B. Pfaff, J. Chow, M. Rosenblum, and D. Boneh. 2003. Terra: A virtual machine-based platform for trusted computing, In Operating Systems Review. ACM SIGOPS Operating Systems Review 37, 193-206. Issue 5.

[13] Github. [n. d.]. Most forked C/C++ repositories. https://github.com/search?l=c\& $\mathrm{p}=1 \& \mathrm{q}=$ stars $\% 3 \mathrm{~A} \% 3 \mathrm{E} 1 \& \mathrm{~s}=$ forks\&type=Repositories.

[14] Johannes Götzfried, Moritz Eckert, Sebastian Schinzel, and Tilo Müller. 2017. Cache Attacks on Intel SGX. In Proceedings of the 10th European Workshop on Systems Security (EuroSec'17). ACM, New York, NY, USA, Article 2, 6 pages. https://doi.org/10.1145/3065913.3065915

[15] J.A. Halderman, S.D. Schoen, N. Heninger, W. Clarkson, W. Paul, J.A. Calandrino, A.J. Feldman, J. Appelbaum, and E.W. Felten. 2008. Lest we remember: Cold boot attacks on encryption keys. In USENIX Security Symposium (SSYM'08). ACM New York, NY, USA, 45-60.

[16] Gernot Heiser and Ben Leslie. 2010. The OKL4 Microvisor: Convergence point of microkernels and hypervisors. In Proceedings of the first ACM asia-pacific workshop on Workshop on systems. ACM, 19-24.

[17] Jorrit N. Herder, Herbert Bos, Ben Gras, Philip Homburg, and Andrew S. Tanenbaum. 2006. MINIX 3: A Highly Reliable, Self-repairing Operating System. SIGOPS Oper. Syst. Rev. 40, 3 (July 2006), 80-89. https://doi.org/10.1145/1151374.1151391

[18] Hewlett-Packard Corporation, Intel Corporation, Microsoft Corporation, Phoenix Technologies Ltd., and Toshiba Corporation. [n. d.]. Advanced Configuration and Power Interface Specification. www.acpi.info/DOWNLOADS/ACPIspec30.pdf.

[19] Matthew Hoekstra, Reshma Lal, Pradeep Pappachan, Vinay Phegade, and Juan Del Cuvillo. 2013. Using innovative instructions to create trustworthy software solutions. In Proceedings of the 2nd International Workshop on Hardware and Architectural Support for Security and Privacy (HASP'13). ACM, New York, NY, USA, 11.

[20] Intel. [n. d.]. Remote Attestation - end to end example. https://software.intel.com/en-us/articles/ intel-software-guard-extensions-remote-attestation-end-to-end-example.

[21] Florian Kelbert, Franz Gregor, Rafael Pires, Stefan Köpsell, Marcelo Pasin, Aurélien Havet, Valerio Schiavoni, Pascal Felber, Christof Fetzer, and Peter Pietzuch 2017. SecureCloud: Secure big data processing in untrusted clouds. In Proceedings of the Conference on Design, Automation \& Test in Europe. European Design and Automation Association, 282-285.

[22] G. Klein, K. Elphinstone, G. Heiser, J. Andronick, D. Cock, P. Derrin, D. Elkaduwe, K. Engelhardt, R. Kolanski, M. Norrish, et al. 2009. seL4: Formal verification of an OS kernel. In Proceedings of the ACM SIGOPS 22nd Symposium on Operating Systems Principles (SOSP'09). ACM, New York, NY, USA, 207-220.

[23] Patrick Koeberl, Steffen Schulz, Ahmad-Reza Sadeghi, and Vijay Varadharajan 2014. TrustLite: a security architecture for tiny embedded devices. In Proceedings of the Ninth European Conference on Computer Systems (EuroSys'14). ACM, New York, NY, USA, 10.
[24] Hugo Krawczyk. 2003. SIGMA: The 'SIGn-and-MAc'approach to authenticated Diffie-Hellman and its use in the IKE protocols. In Annual International Cryptology Conference. Springer, 400-425.

[25] Jaehyuk Lee, Jinsoo Jang, Yeongiin Jang, Nohyun Kwak, Yeseul Choi, Changho Choi, Taesoo Kim, Marcus Peinado, and Brent B Kang. 2017. Hacking in darkness Return-oriented programming against secure enclaves. In USENIX Security. 523539.

[26] Sangho Lee, Ming-Wei Shih, Prasun Gera, Taesoo Kim, Hyesoon Kim, and Marcus Peinado. 2017. Inferring fine-grained control flow inside SGX enclaves with branch shadowing. In 26th USENIX Security Symposium, USENIX Security. 16-18.

[27] Jochen Liedtke. 1996. Toward Real Microkernels. Commun. ACM 39, 9 (1996), 77.

28] Jonathan M. McCune, Yanlin Li, Ning Qu, Zongwei Zhou, Anupam Datta, Virgil Gligor, and Adrian Perrig. 2010. TrustVisor: Efficient TCB Reduction and Attestation. In Proceedings of the IEEE Symposium on Security and Privacy (S\&P'10). IEEE Computer Society, Washington, DC, USA, 143-158

[29] Jonathan M. McCune, Bryan Parno, Adrian Perrig, Michael K. Reiter, and Hiroshi Isozaki. 2008. Flicker: An Execution Infrastructure for TCB Minimization. In Proceedings of the ACM European Conference in Computer Systems (EuroSys'08). ACM, New York, NY, USA, 315-328.

[30] Frank McKeen, Ilya Alexandrovich, Alex Berenzon, Carlos V Rozas, Hisham Shafi, Vedvyas Shanbhogue, and Uday R Savagaonkar. 2013. Innovative instructions and software model for isolated execution. In Proceedings of the 2nd International Workshop on Hardware and Architectural Support for Security and Privacy (HASP'13). ACM, New York, NY, USA, Article 10, 8 pages.

[31] Job Noorman, Pieter Agten, Wilfried Daniels, Raoul Strackx, Anthony Van Herrewege, Christophe Huygens, Bart Preneel, Ingrid Verbauwhede, and Frank Piessens. 2013. Sancus: Low-Cost Trustworthy Extensible Networked Devices with a Zero-Software Trusted Computing Base. In 22nd USENIX Security Symposium (SSYM'13). USENIX Association.

[32] Martin Pool. [n. d.]. distcc: a fast, free distributed C/C++ compiler. https://distcc. github.io/.

[33] C. Priebe. 2018. SGX-LKL. https://github.com/lsds/sgx-lkl.

[34] Ravi Sahita, Ulhas Warrier, and Prashant Dewan. 2009. Protecting Critical Applications on Mobile Platforms. Intel Technology Journal 13, 2 (June 2009), 16-35.

[35] Felix Schuster, Manuel Costa, Cedric Fournet, Christos Gkantsidis, Marcu Peinado, Gloria Mainar-Ruiz, and Mark Russinovich. 2015. VC3: Trustworthy Data Analytics in the Cloud using SGX. In 36th IEEE Symposium on Security and Privacy. IEEE - Institute of Electrical and Electronics Engineers.

[36] Michael Schwarz, Samuel Weiser, Daniel Gruss, Clémentine Maurice, and Stefan Mangard. 2017. Malware Guard Extension: Using SGX to Conceal Cache Attacks. In 14th International Conference on Detection of Intrusions and Malware, and Vulnerability Assessment (DIMVA'17).

[37] Jaebaek Seo, Byoungyoung Lee, Seong Min Kim, Ming-Wei Shih, Insik Shin, Dongsu Han, and Taesoo Kim. 2017. SGX-Shield: Enabling Address Space Layout Randomization for SGX Programs.. In NDSS.

[38] Dmitry Sklyarov. 2017. Intel ME: The Way of Static Analysis. http://blog. ptsecurity.com/2017/04/intel-me-way-of-static-analysis.html.

[39] Raoul Strackx, Pieter Philippaerts, and Frédéric Vogels. 2015. Idea: Towards an inverted cloud. In International Symposium on Engineering Secure Software and Systems. Springer, 111-118.

[40] Raoul Strackx, Frank Piessens, and Bart Preneel. 2010. Efficient Isolation of Trusted Subsystems in Embedded Systems. In Security and Privacy in Communication Networks (SecureComm'10) (Lecture Notes of the Institute for Computer Sciences, Social Informatics and Telecommunications Engineering), Sushil Jajodia and Jianying Zhou (Eds.), Vol. 50. Springer Berlin Heidelberg, 344-361.

[41] R. Ta-Min, L. Litty, and D. Lie. 2006. Splitting interfaces: Making trust between applications and operating systems configurable. In Proceedings of the 7th symposium on Operating systems design and implementation (OSDI'06). USENIX Association, Berkeley, CA, USA, 279-292

[42] Andrew S Tanenbaum and Albert S Woodhull. 1987. Operating systems: design and implementation. Vol. 2. Prentice-Hall Englewood Cliffs, NJ.

[43] Chia-Che Tsai, Donald E Porter, and Mona Vij. 2017. Graphene-SGX: A practical library OS for unmodified applications on SGX. In Proceedings of the USENIX Annual Technical Conference (ATC). 8 .

[44] Jo Van Bulck, Marina Minkin, Ofir Weisse, Daniel Genkin, Baris Kasikci, Frank Piessens, Mark Silberstein, Thomas F Wenisch, Yuval Yarom, and Raoul Strackx. 2018. Foreshadow: Extracting the keys to the Intel SGX kingdom with transient out-of-order execution. In Proceedings of the 27th USENIX Security Symposium. USENIX Association.

[45] Yuanzhong Xu, Weidong Cui, and Marcus Peinado. 2015. Controlled-Channel Attacks: Deterministic Side Channels for Untrusted Operating Systems. In 36th IEEE Symposium on Security and Privacy. IEEE. 